\title{
A Characterization of $k$-Uniform DCSL Graphs
}

\author{
Nageswara Rao K. \\ Department of Mathematics \\ Central University of Kerala \\ Kasaragode, India - 671316.
}

\author{
Germina K.A. \\ Mathematics Research Center \\ Mary Matha Arts \& Science College, Kannur University \\ Mananthavady, India - 670645.
}

\author{
Shaini P. \\ Department of Mathematics \\ Central University of Kerala \\ Kasaragode, India - 671316.
}

\begin{abstract}
Let an injective function $f: V(G) \rightarrow 2^{X}$, where $V(G)$ is the vertex set of a graph $G$ and $2^{X}$ is the power set of a nonempty set $X$, be given. Consider the induced function $f^{\oplus}: V(G) \times$ $V(G) \rightarrow 2^{X} \backslash\{\phi\}$ defined by $f^{\oplus}(u, v)=f(u) \oplus f(v)$, where $f(u) \oplus f(v)$ denotes the symmetric difference of the two sets. The function $f$ is called a $k$-uniform $d c s l$ (and $X$ a $k$-uniform $d c s l$-set) of the graph $G$, if there exists a positive constant $k$ such that $\left|f^{\oplus}(u, v)\right|=k d_{G}(u, v)$, where $d_{G}(u, v)$ is the length of a shortest path between $u$ and $v$ in $G$. If a graph $G$ admits a $k$-uniform dcsl, then $G$ is called a $k$-uniform dcsl graph. In this paper, we initiate a study on 2 -uniform dscl graphs and we establish a characterization for a graph to be $k$-uniform desl.
\end{abstract}

\section{Keywords}

$k$-uniform distance compatible set-labeling, $k$-uniform dcsl index.

\section{Mathematical Subject Classification: 05C78.}

\section{INTRODUCTION}

For all terminology and notation which are not defined in this paper, we refer the reader to F. Harary [3]. Unless mentioned otherwise, all the graphs considered in this paper are finite, simple and without self-loops.

Acharya [1] introduced the notion of vertex set-valuation as a setanalogue of number valuation. For a graph $G=(V, E)$ and a non empty set $X$, Acharya defined a set valuation of $G$ as an injective set valued function $f: V(G) \rightarrow 2^{X}$, and he defined a set-indexer as a set valuation such that the function $f^{\oplus}: E(G) \rightarrow 2^{X} \backslash\{\phi\}$ given by $f^{\oplus}(u v)=f(u) \oplus f(v)$ for every $u v \in E(G)$ is also injective, where $2^{X}$ is the set of all the subsets of $X$ and $\oplus$ is the binary operation of taking the symmetric difference of subsets of $X$.

\section{THEOREM 1. [1] Every graph has a set-indexer.}

\section{DCSL GRAPHS}

Acharya and Germina, who has been studying topological set valuation, introduced the particular kind of set valuation for which a metric, especially the cardinality of the symmetric difference, is associated with each pair of vertices in proportion to the distance between them [2]. In otherwords, the question is whether one can determine those graphs $G=(V, E)$ that admit an injective function $f: V \rightarrow 2^{X}, X$ being a non empty ground set such that the cardinality of the symmetric difference $f^{\oplus}(u v)$ is proportional to the usual path distance $d_{G}(u, v)$ between $u$ and $v$ in $G$, for each pair of distinct vertices $u$ and $v$ in $G$. They called $f$ a distance compatible set labeling (dcsl) of $G$, and the ordered pair $(G, f)$, a distance compatible set labeled (dcsl) graph. Thus

Definition 1. [2] Let $G=(V, E)$ be any connected graph. A distance compatible set labeling (dcsl) of a graph $G$ is an injective set assignment $f: V(G) \rightarrow 2^{X}, X$ being a non empty ground set, such that the corresponding induced function $f^{\oplus}: V(G) \times$ $V(G) \rightarrow 2^{X} \backslash\{\phi\}$ given by $f^{\oplus}(u v)=f(u) \oplus f(v)$ satisfies $\mid$ $f^{\oplus}(u v) \mid=k_{(u, v)}^{f} d_{G}(u, v)$ for every pair of distinct vertices $u, v \in$ $V(G)$, where $d_{G}(u, v)$ denotes the path distance between $u$ and $v$ and $k_{(u, v)}^{f}$ is a constant, not necessarily an integer.

The following universal theorem has been established in [2].

THEOREM 2. 2] Every graph admits a dcsl.

DEFINITION 2. [2] A dcsl $f$ of $G$ is $k$-uniform if all the constant of proportionality with respect to $f$ in Definition 1 are equal to $k$, and if $G$ admits such a dcsl then $G$ is called a k-uniform dcsl graph.

In this paper, we establish a necessary and sufficient condition for a graph to be $k$-uniform dcsl. The remainder of the paper is organized as follows. In Section 2, we study some classes of graphs which admit a 2-uniform dcsl and we calculate the number of induced connected subgraphs, connected subgraphs of a paticular graphs which admit a 2-uniform dcsl. Section 3, we give some defintions about dcsl set-indexer and $k$-uniform dcsl set-indexer and study the behavior of induced subgraph (connected) of a $k$-uniform dcsl graph. Finally, Section 4 provides a characterization for a graph to be $k$ uniform desl graph.

\section{2-UNIFORM DCSL GRAPHS}

In this Section, we first start with a vertex labeling of 2-uniform dcsl graph and prove that every pair of adjacent vertices in a 2uniform dcsl graph $G$ recieve subsets of the same cardinality or the cardinality that differ by 2 .

Proposition 1. Let $(G, f)$ be any 2-uniform dcsl graph, and $u$ and $v$ are adjacent vertices in $G$. Then, either $|f(u)|=|f(v)|$ or $|f(u)|=|f(v)|-2($ or $|f(v)|=|f(u)|-2)$. 
Proof. Suppose $G$ has two adjacent vertices $u$ and $v$, and $f$ be any 2-uniform dcsl of $G$. We prove that either $|f(u)|=|f(v)|$ or $|f(u)|=|f(v)|-2$. Since, by the vertex labeling $f$ of $G$, either $f(u)$ is subset of $f(v)$ or $f(u)$ is not subset of $f(v)$ (similar argument holds, if $u$ replaced by $v$ ).

CASE 1: When $f(u)$ is subset of $f(v)$

Then, $|f(u)|=|f(v)|-2$; otherwise, i.e., if $|f(u)| \neq|f(v)|$ -2 , then $|f(u)|=|f(v)|-i$, where $1 \leq i \neq 2 \leq n$ and $n$ be a finite value. Thus, $|f(u) \oplus f(v)|=i \neq 2 . d(u, v)$, a contradiction.

CASE 2: When $f(u)$ is not subset of $f(v)$

Then, $|f(u)|=|f(v)|$; if not, i.e., $|f(u)| \neq|f(v)|$, then two cases arrises

SUBCASE 2.1: $f(u) \cap f(v)=\emptyset$

Since, $f$ is a 2-uniform dcsl of $G$, and $|f(u)| \neq|f(v)|$, we get $f(u)$ is subset of $f(v)$; a contradiction.

SUBCASE 2.2: $f(u) \cap f(v) \neq \emptyset$

Again, Since, $f$ is a 2-uniform desl of $G$, and $|f(u)| \neq|f(v)|$, here also, we get $f(u)$ is subset of $f(v)$; a contradiction.

Hence, when $f(u)$ is not subset of $f(v)$, then $|f(u)|=|f(v)|$. Thus, in a 2-uniform dcsl graph $G$, if $u$ and $v$ are adjacent in $G$, then, either $|f(u)|=|f(v)|$ or $|f(u)|=|f(v)|-2$.

Let us recall the following definitions related to relations.

(1) $|A|=|B|$, denoted as $R_{1}$, means there is a bijection $A \rightarrow B$.

(2) $|A|<|B|$, denoted as $R_{2}$, means there is an injection $A \rightarrow B$ but no surjection $A \rightarrow B$.

(3) $|A| \leq|B|$, denoted as $R_{3}$, means $|A|<|B|$ or $|A|=|B|$. A partially ordered set (or a poset, in short) $\mathcal{P}$ as a structure $(P, \preceq$ ) where $P$ is a non-empty set and ' $\preceq$ ' is a partial order relation on $P$ such that ' $\preceq$ ' is reflexive, antisymmetric and transitive. We denote $(x, y) \in \mathcal{P}$ by $x \preceq y$. By standard notation, we usually identify the ground set of a poset with the whole poset.

A relation (binary) $R$, on a non-empty set $X$, is an equivalence relation if and only if it is reflexive, symmetric, transitive.

The vertex labeling which is given in Proposition 1 satisfies three interesting properties of relations. Remark 1, Remark 2 and Remark 4 shows the existence of such relations.

REMARK 1. The set $\mathcal{F}$ of vertex labeling of 2-uniform dcsl graph $G$, be such that the adjacent vertices in $G$ recieve subsets of same cardinality, then, $\mathcal{F}$ forms an equivalence relation with respect to $R_{1}$, and forms a poset with respect to set inclusion ' $\subseteq$ '.

The following theorem shows the existence of one such vertex labeling $f$ which is given in Remark 1 to a complete graph.

THEOREM 3. The vertex labeling $f$ of a complete graph $K_{n}$, for $n \geq 2$ admits 2-uniform dcsl, and $|f(u)|=|f(v)|$, for every pair of distinct $u, v \in V\left(K_{n}\right)$.

PRoof. Let $V\left(K_{n}\right)=\left\{v_{1}, v_{2}, . ., v_{n}\right\}$, where $n \geq 2$, and let $X=\{1,2, . ., n\}$.

Define $f: V\left(K_{n}\right) \rightarrow 2^{X}$ by $f\left(v_{i}\right)=\{i\}$, where $1 \leq i \leq n$.

Then, $\left|f\left(v_{i}\right) \oplus f\left(v_{j}\right)\right|=|\{i, j\}|=2 . d\left(v_{i}, v_{j}\right)$, for $1 \leq i \neq j \leq n$. Hence, $f$ is a 2-uniform desl, and also, $\left|f\left(v_{i}\right)\right|=\left|f\left(v_{j}\right)\right|$, for $1 \leq i \neq j \leq n$.

Following the above theorem, we have
COROLlary 1. A graph $G$ with all vertices of full degree is 2-uniform dcsl if and only if $G \cong K_{n}$.

It should note that, the vertex labeling which is given in Theorem 3 is not unique, there exists another vertex labeling $f$ of 2-uniform dcsl $K_{n}$, such that $|f(u)|=|f(v)|-2$, when $u$ and $v$ are adjacent in $V\left(K_{n}\right)$ and one of the vertex $u \in\{u, v\}$, such that $f(u)=\emptyset$. Following result answers this question.

PROPOSITION 2. There exists a vertex labeling $f$ of 2-uniform dcsl $K_{n}, n \geq 2$, such that $f(u)=\emptyset$, for some $u \in V\left(K_{n}\right)$.

PROOF. Let $K_{n}$ be a complete graph on $n$ vertices $v_{1}, v_{2}, \ldots$, $v_{n}, n \geq 2$.

Define $f: V\left(K_{n}\right) \rightarrow 2^{X}$ by $f\left(v_{i}\right)=\{i, n\}$, for $1 \leq i \leq n-1$, and $f\left(v_{n}\right)=\emptyset$. Then, for $1 \leq i \leq n-1,\left|f\left(v_{n}\right)\right|=\left|f\left(v_{i}\right)\right|$ -2 and $\left|f\left(v_{i}\right) \oplus f\left(v_{n}\right)\right|=|\{i, n\}|=2 . d\left(v_{i}, v_{n}\right)$. In particular, $\left|f\left(v_{i}\right) \oplus f\left(v_{j}\right)\right|=|\{i, j\}|=2 . d\left(v_{i}, v_{j}\right)$, for $1 \leq i \neq j \leq n$. Thus, $f$ is a 2-uniform desl.

Now, we consider the set of vertex labeling of 2-uniform dcsl of $G$, such that the adjacent vertices in $G$ recieve subsets of the cardinality that differ by 2 and it is easy to prove that it forms a poset with respect to set inclusion ' $\subseteq$ '.

REMARK 2. The set $\mathcal{F}$ of vertex labeling of 2-uniform dcsl graph $G$, such that the adjacent vertices in $G$ recieve subsets of the cardinality that differ by 2 , then, $\mathcal{F}$ forms a poset with respect to set inclusion ' $\subseteq$ '.

We can prove that $K_{1, n}$, where $n \geq 1$ admits the vertex labeling of 2-uniform dcsl which is discussed in Remark 2. Hence we have the following theorem.

THEOREM 4. The vertex labeling $f$ of a star graph $K_{1, n}$, where $n \geq 2$ admits 2-uniform dcsl, and for each pair of adjacent vertices in $K_{1, n}$, $f$ recieve subsets of the cardinality that differ by 2 .

PROOF. Let $\left\{v_{0}, v_{1}, v_{2}, . ., v_{n}\right\}$, where $n \geq 2$ be the vertices of $K_{1, n}$ with $v_{0}$ as its center, and let $X=\{1,2, . ., 2 n\}$.

Define $f: V\left(K_{1, n}\right) \rightarrow 2^{X}$ by $f\left(v_{0}\right)=\emptyset$, and $f\left(v_{i}\right)=\{i, i+n\}$, where $1 \leq i \leq n$.

Then, for $i \geq 1\left|f\left(v_{0}\right) \oplus f\left(v_{i}\right)\right|=|\{i, i+n\}|=2 . d\left(v_{0}, v_{i}\right)$, and $\left|f\left(v_{i}\right) \oplus f\left(v_{j}\right)\right|=|\{i, i+n, j, j+n\}|=2 . d\left(v_{i}, v_{j}\right)$, for $1 \leq i \neq j \leq n$. Hence, $f$ is a 2-uniform dcsl.

Finally, we prove that $|f(u)|=|f(v)|-2$ (or $|f(v)|=|f(u)|$ -2 ), when $u$ and $v$ are adjacent in $V\left(K_{1, n}\right)$. Since, for $1 \leq i \leq n$, only $v_{0}$ is adjacent to each $v_{i}$ in $V\left(K_{1, n}\right)$, thus $\left|f\left(v_{0}\right)\right|=\left|f\left(v_{i}\right)\right|$ -2 .

REMARK 3. In the above theorem, we may assign the vertex labeling $f(u)=\emptyset$, to any one of the vertex $u \in V\left(K_{1, n}\right)$, then also, $K_{1, n}$ admits 2-uniform dcsl (See Figure 1).

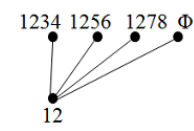

Fig. 1. 2-uniform des1 Vertex labeling of $K_{1, n}, n \geq 2$. 
Finally, we consider the set of vertex labeling of 2-uniform dcsl of $G$, such that the adjacent vertices in $G$ receive subsets of same cardinality or the cardinality that differ by 2 , then we have the following remark.

REMARK 4. Let $\mathcal{F}$ be the range of a vertex labeling of 2-uniform $d c s l$ graph $G$, then, $\mathcal{F}$ does not form neither a poset nor an equivalence relation with respect to $R_{3}$ but it forms a poset with respect to set inclusion ' $\subseteq$ '.

REMARK 5. In Theorem 4, we could observe the vertex labeling $f$ which is same as the vertex labeling $f$ that is given in Remark 4 and we have either $|f(u)|=|f(v)|$ or $|f(u)|=|f(v)|-2$ (or $|f(v)|=|f(u)|-2)$, when $u$ and $v$ are distinct pair of vertices in $K_{1, n}$.

One should note that every subgraph of a 2-uniform desl graph $G$ need not be connected. Thus we are interested to test the subgraphs (connected) of a 2-uniform dcsl graph $G$, whether it admits 2-uniform dcsl or not. The following results tell about the induced subgraphs of a 2-uniform dcsl complete graph $K_{n}$.

Proposition 3. Every induced subgraph of a complete graph $K_{n}$ admits 2-uniform dcsl.

PROOF. Since every induced subgraph of a complete graph is complete, and by Theorem 3 these complete graphs admits 2uniform desl.

The following theorem shows the number of induced subgraphs of a complete graph that admits 2-uniform dcsl. For the completeness of the statement we are including null graph (a graph with out vertices) and trivial graph (a graph with only one vertex) which are clearly 2-uniform dcsl graphs.

THEOREM 5. The number of induced subgraphs of a complete graph $K_{n}$ that admits 2-uniform dcsl is $2^{\left|V\left(K_{n}\right)\right|}$.

PROOF. Since by Proposition 3, each induced subgraph of $K_{n}$ is 2-uniform dcsl. Thus every subgraph $G[H]$, induced by $H \subseteq$ $V\left(K_{n}\right)$ admits 2-uniform dcsl. Since the order of $K_{n}$ is $n$. Hence, we get $2^{\left|V\left(K_{n}\right)\right|}$ number of induced subgraphs that admits 2uniform dcsl.

REMARK 6. In the above Theorem 5, if we restrict to non-trivial (a graph with two or more vertices) induced subgraphs, then the number of non-trivial induced subgraphs of a complete graph $K_{n}$ that admits 2-uniform dcsl is $2^{\left|V\left(K_{n}\right)\right|}-\left|V\left(K_{n}\right)\right|-1$.

Since the graphs $K_{n}$ and $K_{1, n}$ are admitting 2-uniform dcsl, so it is clear that the connected subgraphs of a complete graph $K_{n+1}$ which always admit 2-uniform dcsl are $K_{1, r}$, where $1 \leq r \leq n$. Thus, to find the number of connected subgraphs of $K_{n+1}$ which is of the form $K_{1, r}$, where $1 \leq r \leq n$ is an interesting problem.

THEOREM 6. Let $G$ a complete graph on $n+1$ vertices, then the number of 2-uniform dcsl connected subgraphs of $G$ which is of the form $K_{1, r}$, where $1 \leq r \leq n$ is $|V(G)|\left(2^{|V(G)|-1}-\right.$ $\left.\frac{|V(G)|-1}{2}-1\right)$.

Proof. Suppose $G \simeq K_{n+1}$. Since by Theorem 4 , for $1 \leq$ $r \leq n, K_{1, r}$ is 2-uniform dcsl, so it suffices to prove this theorem by counting individually the number of subgraphs of the form
$K_{1, n}, K_{1,(n-1)}, K_{1,(n-2)}, \ldots, K_{1,1}$ and adding this number we get the required result. We first count the number of subgraphs of the form $K_{1, n}$. Since they are $n+1$ number of full degree vertices in $G$ and each of these vertices including with their corresponding neighboring vertices forms a connected subgraph of the form $K_{1, n}$, hence they are $n+1$ number of connected subgraphs of the form $K_{1, n}$. Secondly, to get the number of connected subgraphs of the form $K_{1,(n-1)}$, we remove one leaf from $K_{1, n}$ out of $n$ leaves, so they are $n_{c_{1}}$ ways to choose the number of graphs of the form $K_{1,(n-1)}$. Since they are $n+1$ of such $K_{1, n}$, hence, the total number of connected subgraphs of the form $K_{1,(n-1)}$ is $n_{c_{1}}(n+1)$. In the same way, removing 2 leaves from $K_{1, n}$ out of $n$ leaves, yields $n_{c_{2}}(n+1)$ number of subgraphs of the form $K_{1,(n-2)}$. Repeating this process up to the removal of $n-2$ leaves and the removal of $n-2$ leaves, yields $n_{c_{n-2}}(n+1)$ number of subgraphs of the form $K_{1,2=(n-(n-2))}$. Lastly, removing $n-1$ leaves from each $K_{1, n}$ is equal to the number of edges in $G$, which is equal to $(n+1)_{c_{2}}$. Thus, by adding all the number of subgraphs of the form $K_{1, n}$, $K_{1,(n-1)}, K_{1,(n-2)}, \ldots, K_{1,1}$ together, we get the total number of 2uniform dcsl connected subgraphs of $G$ which is of the form $K_{1, r}$, where $1 \leq r \leq n$ is $(n+1)\left(n_{c_{0}}+n_{c_{1}}+. .+n_{c_{n-2}}\right)+(n+1)_{c_{2}}=$ $(n+1)\left(2^{n}-n-1\right)+\frac{n(n+1)}{2}=(n+1)\left(2^{n}-\frac{n}{2}-1\right)$ $=|V(G)|\left(2^{s}-\frac{s}{2}-1\right)$

where $s=|V(G)|-1$

From Theorem 6, we immediately get an analogous result for the number of non-trivial connected subgraphs of $K_{1, n}$.

PROPOSITION 4. The number of non-trivial connected subgraphs of a star graph $K_{1, n}$ that admits 2-uniform dcsl is $2^{\left|V\left(K_{1, n}\right)\right|}-1$

\section{SET-INDEXER OF A DCSL GRAPH}

Since induced subgraphs are not always connected, thus, every induced subgraph of a 2-uniform dcsl graph $G$ need not be 2-uniform dcsl. Now, it is interesting to know the behavior of connected induced subgraph of a 2-uniform desl graph $G$, whether it admits 2-uniform dcsl or not, for that, we are defining dcsl set-indexer and $k$-uniform desl set-indexer as follows.

Definition 3. Let $(G, f)$ be a dcsl graph. A dcsl set-indexer (DSI, in short) of $G$ is an injective function $f$ such that the induced function $f^{\oplus}$ on $E(G)$ is also injective.

Since, by Definition 1 of dcsl graph $(G, f)$, it is not sure that every induced function $f^{\oplus}$ of $f$ is an injective function on $V(G) \times V(G)$. Thus, we have

THEOREM 7. For a dcsl graph $(G, f)$, if the constants of proportionality $k_{(u, v)}^{f}, u \neq v \in V(G)$ are all distinct. Then, the induced function $f^{\oplus}$ of $f$ is an injective function on $V(G) \times V(G)$.

Proof. Suppose $G$ be a graph and $f$ be a desl labeling of $G$. Since by definition of dcsl, to each pair $u \neq v \in V(G)$, there exists a constant of proportionality $k$ associated with $f$. Now, if this $k$ 's are different means the labeling $f(u) \oplus f(v)$ associated with $k$ are become different, which means that $f^{\oplus}$ is injective.

The converse of the above theorem may not be true always, i.e., there exists graphs whose edge labeling is injective, but the constants of proportionality are not distinct (See Figure 2). 


\section{$\stackrel{12}{\mathrm{k}=2} \underset{\mathrm{k}=2}{\mathbf{3}}$}

Fig. 2. 2-uniform desl Vertex labeling of $P_{3}$.

Definition 4. [6] A dcsl $f$ is dispersive if the constants of proportionality $k_{(u, v)}^{f}, u \neq v \in V(G)$ are all distinct and $G$ is dispersive if it admits a dispersive dcsl.

A dcsl $f$ of $a(p, q)$-graph $G$ is edge-dispersive if the constants of proportionality $k_{(u, v)}^{f}, u \neq v \in E(G)$ are all distinct and $G$ is edge-dispersible graph if it admits an edge-dispersive dcsl.

THEOREM 8. Every dispersive dcsl graph $(G, f)$ admits DSI.

PROOF. Since $f$ is dispersive of $G$, so that all the constants of proportionality $k_{(u, v)}^{f}, u \neq v \in V(G)$ are all distinct and by Theorem 7. the induced function $f^{\oplus}$ of $f$ is an injective function on $V(G) \times V(G)$, hence $f^{\oplus}$ is an injective on $E(G)$, and hence $G$ admits DSI.

Since all dispersible dcsl-graphs are edge-dispersible. Thus invoking Theorem 8

THEOREM 9. Every edge-dispersive dcsl graph $(G, f)$ admits DSI.

DEFINITION 5. [6] A dcsl $f$ of a graph $G$ is $(k, r)$-arithmetic if the constants of proportionality with respect to $f$ can be arranged in the arithmetic progression as $k, k+r, k+2 r, \ldots, k+(q-1) r$ and if $G$ admits such a dcsl then, $G$ is a $(k, r)$-arithmetic dcsl-graph.

THEOREM 10. Every $(k, r)$-arithmetic dcsl graph $(G, f)$ admits DSI.

Proof. Since by definition of $(k, r)$-arithmetic dcsl graph $(G, f)$, we have all the constants of proportionality $k_{(u, v)}^{f}, u \neq$ $v \in E(G)$ are all distinct, which means the edge labeling $f^{\oplus}(e)$ is different for each $e \in E(G)$, hence $G$ admits DSI.

Definition 6. [6] Let $(G, f)$ be a k-uniform dcsl graph. A $k$ uniform dcsl set-indexer ( $k$-UDSI, in short) of $G$ is an injective function $f$ such that the induced function $f^{\oplus}$ on $E(G)$ is also injective, and $\left|f^{\oplus}(e)\right|=k$ for all $e \in E(G)$, where $k$ be a positive integer.

Since, by Definition 2 of $k$-uniform dcsl graph $(G, f)$, it is not sure that every induced function $f^{\oplus}$ of $f$ is an injective function on $V(G) \times V(G)$, so that for a $k$-uniform dcsl of $G$, it is not guarantee that $G$ admits $k$-UDSI, but depends on $k$-uniform vertex labeling $f$ of $G$, they are graphs which admits $k$-UDSI. Thus, we have

THEOREM 11. If the induced function $f^{\oplus}$ of $f$, of a $k$-uniform $d c s l$ graph $(G, f)$, is an injective function on $V(G) \times V(G)$. Then, $G$ admits k-UDSI.

THEOREM 12. If $G$ has 2-uniform dcsl $f$, such that $|f(u)|=\mid$ $f(v) \mid$, for every pair of distinct $u, v \in V(G)$. Then, G has 2-UDSI.

Proof. We have already proved in Theorem 2 that there exists a graph $G$ whose vertex labeling $f$ satisfies $|f(u)|=|f(v)|$, for every pair of distinct $u, v \in V(G)$.
Suppose $G$ be a graph on $n$ vertices $v_{1}, v_{2}, . ., v_{n}$ and it has a 2uniform dcsl $f$ which is given in Theorem 2 Then, for $1 \leq i \neq$ $j \leq n,\left|f\left(v_{i}\right)\right|=\left|f\left(v_{j}\right)\right|$, and $f^{\oplus}\left(v_{i}, v_{j}\right)=\{i, j\}$, which is an injective function, hence, by Theorem 11 , for $k=2, G$ has 2-UDSI.

The converse of the above theorem need not be true always, i.e., there exists 2-uniform dcsl graphs which admits 2-UDSI such that the adjacent vertices in $G$ recieve subsets of the cardinality that differ by 2 (See Figure 1).

Now, we are trying to answer the question whether connected induced subgraph of a 2-uniform dcsl graph $G$, admits 2-uniform dcsl or not.

THEOREM 13. If $G$ has 2-uniform dcsl $f$, such that $|f(u)|=\mid$ $f(v) \mid$, for every pair of distinct $u, v \in V(G)$. Then, any connected induced subgraph $H$ of $G$ admits 2-uniform dcsl.

Proof. Let $G$ be a graph of order $n$ which has a 2-uniform dcsl $f$, such that $|f(u)|=|f(v)|$, for every $u \neq v \in V(G)$, then by Theorem 12 $G$ admits 2-UDSI, which means the induced function $f^{\oplus}$ of $f$ is an injective function on $E(G)$.

Let $H$ be a connected induced subgraph of $G$ and $f^{\star}$ be the restriction of $f$ to $V(H)$. Then, $f_{f^{\star}}^{\oplus}$ is the corresponding restriction of $f^{\oplus}$ to $V(H) \times V(H)$.

Thus, clearly, $f_{f^{\star}}^{\oplus}$ is an injective function on $V(H) \times V(H)$ and for every $u \neq v \in V(H),\left|f^{\star}(u)\right|=\left|f^{\star}(v)\right|$ and $\left|f_{f^{\star}}^{\oplus}(u, v)\right|=$ $2=2 . d(u, v)$; otherwise there exists $p \neq q \in V(H)$, such that $\left|f^{\star}(p)\right| \neq\left|f^{\star}(q)\right|$ and $\left|f^{\oplus}(p q)\right| \neq 2$; a contradiction (since $G$ has 2-UDSI and $|f(u)|=|f(v)|$, for every $u \neq v \in V(G)$ ). Hence, $H$ has 2-uniform desl.

Invoking Theorem 13 we have

COROLLARY 2. If $G$ has 2-uniform dcsl $f$, such that $|f(u)|=\mid$ $f(v) \mid$, for every pair of distinct $u, v \in V(G)$. Then, any connected induced subgraph $H$ of $G$ admits 2-UDSI.

REMARK 7. There are some 2-uniform dcsl graphs $G$ such that the adjacent vertices in $G$ recieve subsets of the cardinality that differ by 2 and the connected induced subgraphs of $G$ admits 2uniform dcsl (See Figure 3).

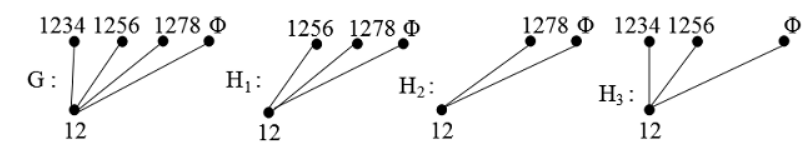

Fig. 3. The 2-uniform desl of $G$, and its connected induced subgraphs $H_{1}$, $\mathrm{H}_{2}$ and $\mathrm{H}_{3}$.

Generalizing Theorem 13 for a $k$-uniform dcsl graph $G$, we get

THEOREM 14. If the induced function $f^{\oplus}$ of $f$, of a $k$-uniform dcsl graph $(G, f)$, is an injective function. Then, any connected induced subgraph $H$ of $G$ admits $k$-uniform dcsl.

By Theorem 14, the following is obtained as its analogous result.

COROLlaRY 3. If the induced function $f^{\oplus}$ of $f$, of a $k$-uniform dcsl graph $(G, f)$, is an injective function. Then, any connected induced subgraph $H$ of $G$ admits $k$-UDSI. 


\section{NON- $K$-UNIFORM DCSL GRAPH}

In this section, we start with the non- $k$-uniform desl graph (a graph in which there is no possibility of the existence of any vertex labeling of $k$-uniform dcsl) and prove that a graph is non- $k$-uniform $\mathrm{dcsl}$, if it contains a non- $k$-uniform dcsl subgraph. Using the existence of non- $k$-uniform dcsl graph condition, we able to prove the necessary and sufficient condition for $k$-uniform dcsl graph.

THEOREM 15. If a graph $G$ contains a non- $k$-uniform dcsl as connected subgraph, then that graph $G$ is non- $k$-uniform dcsl.

PROOF. We prove by contrapositive, i.e., every connected subgraph of a $k$-uniform dcsl graph $G$ is a $k$-uniform dcsl. Suppose $G$ be a graph and $f$ be a $k$-uniform dcsl of $G$. We prove this theorem in 2 cases.

CASE 1: If the induced function $f^{\oplus}$ of $f$ is an injective function, then by Theorem 14 every connected subgraph $H$ of $G$ admits $k$ uniform desl.

CASE 2: If the induced function $f^{\oplus}$ of $f$ is not an injective function, then every connected subgraph $H$ of $G$ need not be $k$-uniform dcsl but it always possible to consider another vertex labeling to $H$ which admits $k$-uniform dcsl (since the given graph $G$ is $k$-uniform $\mathrm{dcsl}$ and the existence of vertex labeling of $k$-uniform desl is not unique).

Now, we prove the necessary and sufficient condition for a graph $G$ to be $k$-uniform desl.

THEOREM 16. A graph $G$ (connected) admits $k$-uniform dcsl if and only if $G$ does not contain any non- $k$-uniform $d c s l$ as a subgraph.

Proof. Suppose $G$ admits $k$-uniform dcsl. If possible, suppose $G$ contains a non- $k$-uniform dcsl as subgraph, then by Theorem 15 . $G$ is non- $k$-uniform desl, which is a contradiction.

Conversely, if a graph $G$ does not contain any non- $k$-uniform dcsl as a subgraph, which is equivalent to saying that every subgraph (connected) of a graph $G$ is $k$-uniform desl, then obviously $G$ is $k$-uniform desl (since, by hypothesis, $G$ is itself a subgraph which is $k$-uniform desl).

\section{CONCLUSION}

From Theorem 15 we can prove a given graph $G$ is non- $k$-uniform $\mathrm{dcsl}$, when it contains a non- $k$-uniform dcsl as connected subgraph. Also, by Theorem 16, we can say that every subgraph (connected) of a graph $G$ is $k$-uniform desl, when $G$ is $k$-uniform desl.

\section{ACKNOWLEDGMENTS}

The authors are thankful to the Department of Science and Technology, Government of India, New Delhi, for the financial support concerning the Major Research Project (Ref: No. SR/S4/MS : 760/12).

\section{REFERENCES}

[1] Acharya, B. D., 1983, Set-valuations of Graphs and Their Applications. MRI Lecture Notes in Applied Mathematics, No.2, Mehta Research Institute of Mathematics and Mathematical Physics, Allahabad.
[2] Acharya, B. D., and Germina, K. A., 2011, Distance compatible Set-labeling of Graphs, Indian J. Math. and Comp. Sci. Jhs., 1, 49 - 54.

[3] Harary, F., 1969, Graph theory, Addison wesley publ. comp. Reading, Massachusetts.

[4] Germina, K. A., 2012, Uniform Distance-compatible Setlabelings of Graphs, J. of Combinatorics, Information and System Sciences, Vol. 37, 169-178.

[5] Germina, K. A., and Thomas, B. K., 2011, Distance Compatible Set-Labeling of Graphs, International Mathematical Forum, Vol. 6(31), 1513-1520.

[6] Thomas, B. K., and Germina, K. A., 2010, $(k, r)$-Distance Compatible Set-Labeling of Graphs, International Mathematical Forum, Vol. 5(45), 2237-2247.

[7] Germina, K. A., and Nageswararao, K., 2015, Characterization of Vertex labeling of 1- uniform dcsl graph which form a lattice, Journal of Fuzzy Set Valued Analysis, Vol. 2, 166-170.

[8] Nageswara Rao, K., and Germina, K. A., 2015, Dimension of Vertex Labeling of $k$ - uniform dcsl path, Advances and Applications in Discrete Mathematics, to appear. 